\title{
Parametric Identification of a Base-Excited Single Pendulum
}

\author{
YANG LIANG and B. F. FEENY \\ Department of Mechanical Engineering, Michigan State University, East Lansing, MI 48824, USA
}

\begin{abstract}
A harmonic balance based identification algorithm was applied to the simulated single pendulum with horizontal base-excitation. The purpose of this simulation was to examine the applicability of the algorithm on parametrically excited, whirling chaotic systems. Modifications were adopted to adapt to the whirling systems. The system was supposed to be unknown except only the excitation frequency. Linear interpolation functions and the Fourier series functions were tested to approximate unknown nonlinear functions in the governing differential equation. After extracting unstable periodic orbits, all of the parameters were simultaneously identified. By direct comparison, Poincaré section plots and reconstructed phase portrait techniques, it was shown that the identified system had similar dynamical characteristics to the original simulated pendulum, which implies the effectiveness of the examined algorithm.
\end{abstract}

Keywords: parametric identification, harmonic balance method, chaotic systems, unstable periodic orbits, whirling systems, pendulums.

\section{Introduction}

A simulated, horizontally base-excited pendulum is investigated for chaotic system identification. A harmonic balance parametric identification method is examined here, because of its simplicity and capacity of handling chaotic data [26].

Pendulum systems are among the most thoroughly investigated dynamic systems in chaotic, nonlinear system research (references [1-7], etc. ), for their simplicity in both theoretical expression and experimental validation. Water [1] studied the unstable periodic orbits of a vertically excited system. Jeong and Kim [2] investigated the bifurcation phenomena and routes to chaos of a horizontally excited system. Furthermore, Bishop [3] and Dooren [4] studied the parametric regions of chaos of a parametrically excited pendulum.

Parametric identification of nonlinear systems has seen a lot of progress in recent years. Direct identification method [8] can be applied provided that all the displacement, velocity and acceleration signals are known or can be obtained. However, it is usually not so in applications, and the method is vulnerable to noise contamination. Chen and Tomlinson [9] developed an efficient AVD time series model, which accommodates the acceleration, velocity and displacement (AVD) simultaneously by means of the fast Fourier transform. Plakhtienko $[10,11]$ introduced a method of special weight functions, by which the second order differential equation could be converted to a series of linear equations if some periodic orbits are known. Hence, the differential equation can be transformed into matrix equations. Like many other studies (references [9-20]), these methods

(C) 2005 Kluwer Academic Publishers. Printed in the Netherlands. 
focused on two types of identification procedures: random/impulse excitation response or forced steady state periodic vibration. Furthermore, the random/impulse excitation methods based upon the FFT and/or the frequency response function [19, 20] actually apply to weak nonlinearity due to their assumptions. Nonlinear free vibration was also investigated for identification purposes. Feldman [21, 24] introduced a method of utilizing the Hilbert transformation to free vibration systems. But, it is unclear whether the Hilbert transform method can be applied to multi-degreeof-freedom systems. Ghanem [25] proposed an algorithm based on wavelet analysis. The algorithm was tested on single and multi-degree of freedom (d. o. f. ) systems, and was proven effective for free and forced non-chaotic vibration. The wavelet analysis method is based upon the orthogonality of the wavelet basis functions, it is thus essentially similar to the harmonic balance method, which utilizes the orthogonality of the Fourier series orthonormal functions. The wavelet basis functions are time localized, while the harmonic basis functions apply to the entire duration of time. Recently, a method [22, 23] which exploits proper orthogonal decomposition (POD) and optimization was developed for nonlinear systems and tested on simple chaotic systems. Meanwhile, based upon the harmonic balance method [15-18], Feeny and Yuan [26, 27] proposed a method for chaotic systems, which also exploits the extracted unstable periodic orbits. This method was successfully applied to single degree of freedom systems, and is theoretically applicable to chaotic or non-chaotic, strongly nonlinear multi-degree of freedom systems. Thus, this study is to present new research results by applying the method to more complicated chaotic systems. As we know, the harmonic balance method requires periodic responses for identification purposes. For linear systems, the method can be simplified to frequency response functions (FRF) method if periodic responses can be obtained at multiple frequencies. For non-chaotic, nonlinear systems, the harmonic balance method also have advantages over other methods due to its simple algorithm and/or the resistance to noise contamination. For chaotic systems, the chaotic response is no-periodic. However, for hyperbolic chaos, if unstable periodic orbits (UPOs) lie within, we can extract the approximated unstable periodic orbits from the chaotic data. Hence, the harmonic balance identification can be applied based upon the UPOs. On the other hand, many other identification methods can not be applied to chaotic systems. Noticeably, the wavelet analysis method [25] can also be applied for chaotic system since both the wavelet method and the harmonic balance method are based upon orthonormal basis and signal reconstruction. The harmonic balance method has simpler algorithm and is easier for industry applications. The wavelet analysis, on the other hand, is more suitable for identification of the time-variant parameters. Since our present study focused on systems with time-invariant parameters, the harmonic balance method is a more suitable choice. 
Our purpose here is to apply the harmonic balance algorithm to the horizontally excited pendulum system such that we can examine the algorithm applicability on systems with chaotic whirling behavior and strong nonlinearity. Also, the single pendulum was investigated due to its simplicity in simulation and experiment verification. The identification method has been examined on smooth excitation single d. o. f. systems with simple nonlinearity [26]. However, the pendulum is a more complicated case, and the algorithm must be modified. In the following sections, the single pendulum system and the modified method will be introduced first. Pre-requirements of applying this method will be discussed, primarily regarding the phase plane reconstruction. In the last two sections, the simulation results will be presented and discussed.

\section{Horizontally excited single pendulum \& identification algorithm}

Single pendulum systems have simple structures, but strong non-linearity due to their whirling property. The governing differential equations can be simulated easily, and the experimental verification is also feasible. Based upon these advantages, horizontally base excited single pendulums are chosen for investigation here. The non-dimensional form of the differential equation is

$$
\ddot{\theta}+2 \xi / r \dot{\theta}+1 / r^{2} \sin \theta-f \sin t \cos \theta=0
$$

where $t=\omega \tau, \omega$ is the angular excitation velocity, and $\tau$ is the actual time; $\xi$ is the viscous

damping coefficient; $r=\omega / \omega_{N}, \omega_{N}=\sqrt{\frac{m g e}{J}}$ is the natural frequency of the linearized system, $m$ is the pendulum mass, $e$ is the the centroid offset from the pendulum hinge, $J$ is the moment of inertia about the hinge point. Meanwhile coefficient $f=\frac{m e}{J} a$, and $a$ is the excitation amplitude. For simplicity, we denote $c_{r}=2 \xi / r$ as the new non-dimensional friction coefficient. The function $f \sin t \cos \theta$ is the nonlinear parametric excitation term, and $1 / r^{2} \sin \theta$ is the autonomous nonlinear part. The angular displacement $\theta$ is in $\left.S^{[}-\pi, \pi\right) 1$ space (one dimensional sphere space), whereas the angular velocity and acceleration are in $R^{1}$ space.

To apply the identification algorithm [26] to the pendulum system, the following more general expression of (1) can be assumed:

$$
c_{r} \dot{x}+k x+f_{\text {anl }}(x, \dot{x})+\left\{d_{1}+f_{\text {pnl }}(x, \dot{x})\right\} p(t)=-\ddot{x},
$$

where $k$ is the linear stiffness parameter, $f_{a n l}(x, \dot{x})$ is the autonomous nonlinear part, $f_{p n l}(x, \dot{x})$ is the time-independent part of the parametric excitation term, and coefficient $d_{1}$ is the amplitude of external excitation force. If $d_{1}$ is non-zero and $f_{p n l}$ equal to zero, the system becomes a nonlinear system with external excitation force. On the contrary, if $d_{1}$ is zero and $f_{p n l}$ is non-trivial, it is 
then a parametrically excited system. For the examined single pendulum, parameter $k=0$ and $d_{1}=0$. Meanwhile, since the non-linearity of the pendulum is caused by geometry, it is convenient to assume $f_{a n l}$ and $f_{p n l}$ in equation (2) as functions of only displacement, such that

$$
c_{r} \dot{x}+f_{a n l}(x)+f_{p n l}(x) p(t)=-\ddot{x} .
$$

Based on equation (3), it is assumed that nothing is known a priori except the harmonic excitation term $p(t)=\alpha_{1} \cos \omega t+\beta_{1} \sin \omega t$. Unknown functions $f_{p n l}$ and $f_{\text {anl }}$ can be approximated by

$$
\begin{aligned}
f_{p n l} & \approx \sum_{i=1}^{N} P_{i} \phi_{i}(x), \\
f_{\text {anl }} & \approx \sum_{i=1}^{N} q_{i} \phi_{i}(x),
\end{aligned}
$$

where $\left\{\phi_{i}(x)\right\}$ is a set of basis functions, and $P_{i}, q_{i}$ are unknown parameters. Since the choice of basis functions can affect accuracy of the identified functions, two sets of basis functions were tested in the simulation: linear interpolation functions and Fourier series functions.

Substituting (4), (5) and harmonic excitation into (3), we have

$$
c_{r} \dot{x}+\sum_{i=1}^{N} q_{i} \phi_{i}(x)+\sum_{i=1}^{N} n_{i} \phi_{i}(x) \cos t+\sum_{i=1}^{N} p_{i} \phi_{i}(x) \sin t=-\ddot{x},
$$

where $n_{i}=P_{i} \alpha_{1}$ and $p_{i}=P_{i} \beta_{1}$. With equation (6), harmonic balance identification [26] can be applied after extracting unstable or stable periodic orbits from the collected displacement data. For a period $k$ orbit, if $x$ in $R^{1}$ (one dimensional real space), the displacement, velocity and acceleration signals can be approximated by the following truncated Fourier series expansions:

$$
\begin{aligned}
& x_{k}(t) \approx \frac{a_{0, k}}{2}+\sum_{j=1}^{M}\left(a_{j, k} \cos \frac{j \omega t}{k}+b_{j, k} \sin \frac{j \omega t}{k}\right), \\
& \dot{x}_{k}(t) \approx \sum_{j=1}^{M} \frac{j \omega}{k}\left(-a_{j, k} \sin \frac{j \omega t}{k}+b_{j, k} \cos \frac{j \omega t}{k}\right), \\
& \ddot{x}_{k}(t) \approx \sum_{j=1}^{M}-\frac{j^{2} \omega^{2}}{k^{2}}\left(a_{j, k} \cos \frac{j \omega t}{k}+b_{j, k} \sin \frac{j \omega t}{k}\right),
\end{aligned}
$$

where, for non-dimensional equation (6), $\omega=1$. However, for $x$ in $S^{1}$ (one dimensional sphere space, such as angular displacement $\theta$ in the single pendulum), Equations (7) - (9) are not applicable. The harmonic balance method can still be applied here, but a modification is needed to derive the velocity and acceleration $R^{1}$ signals from the sampled $S^{1}$ displacement signal. 


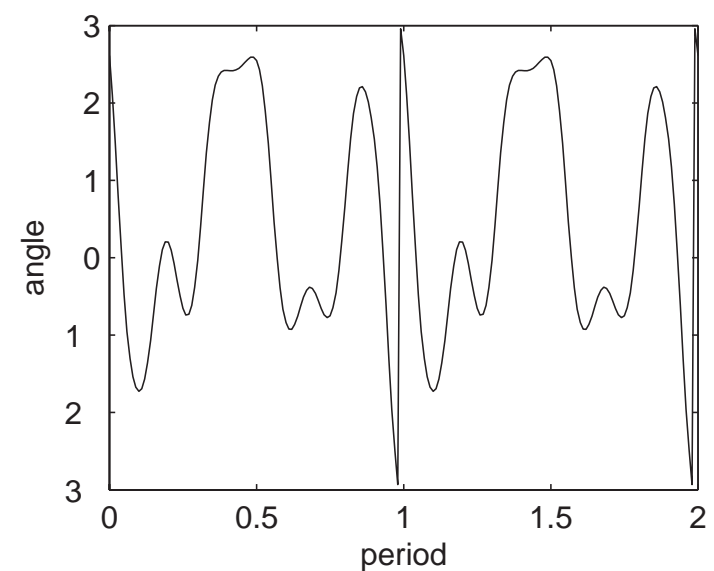

(a)

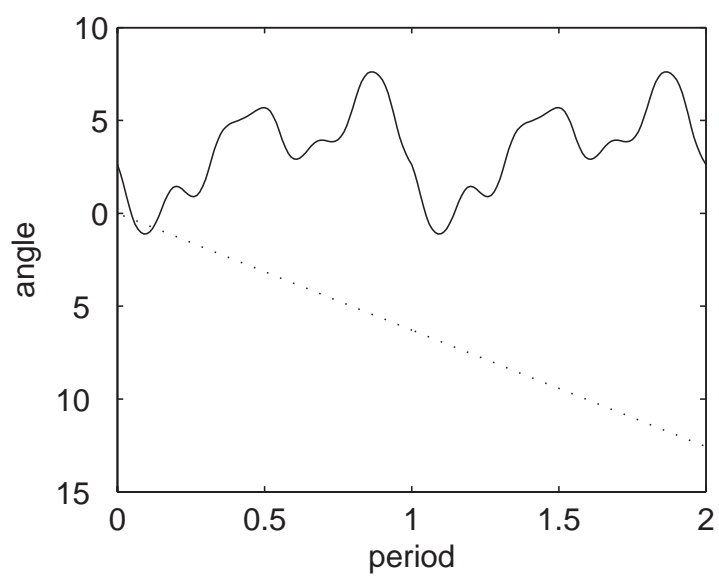

(c)

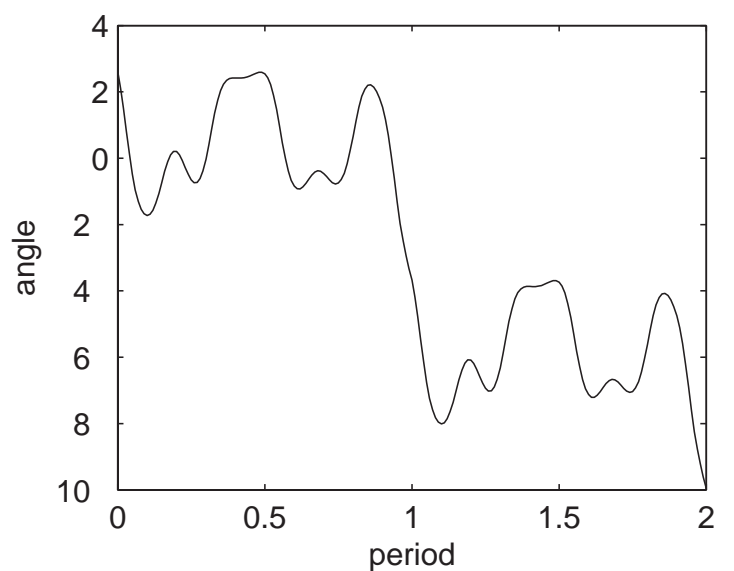

(b)

Figure 1. A period-4 orbit (a) sampled signal; (b) actual continuous signal $x_{c} ;$ (c) $\cdots$ constant rotation part $\omega_{c k} t,-$ oscillatory part $x_{v a r}$.

Consider an experiment in which whirling angle $\theta$ is sensed with an encoder such that the output $x$ has values in the interval $[-\pi, \pi)$. Thus, $x=\operatorname{\theta mod}(2 \pi)-\pi$ is discontinuous. According to the illustration in Figure 1 of a period four orbit of the single pendulum system, the sampled data $\{x\}$ should be converted to a continuous signal $\left\{x_{c}\right\}$ in $R^{1}$, and then decomposed as

$$
x_{c k}=\omega_{c k} t+x_{v a r, k},
$$

where $\omega_{c k} t$ represents the constant rotating part of a whirling periodic orbit, and $x_{v a r, k}$ is the oscillatory part of the orbit signal, which can be approximated by the truncated Fourier series expansion

$$
x_{v a r, k}(t) \approx \frac{u_{0, k}}{2}+\sum_{j=1}^{M}\left(u_{j, k} \cos \frac{j \omega t}{k}+v_{j, k} \sin \frac{j \omega t}{k}\right) .
$$


Based upon $x_{c k}$, the corresponding velocity and acceleration can be expressed as

$$
\begin{gathered}
\dot{x}_{k}(t) \approx \omega_{c k}+\sum_{j=1}^{M} \frac{j \omega}{k}\left(-u_{j, k} \sin \frac{j \omega t}{k}+v_{j, k} \cos \frac{j \omega t}{k}\right), \\
\ddot{x}_{k}(t) \approx \sum_{j=1}^{M}-\frac{j^{2} \omega^{2}}{k^{2}}\left(u_{j, k} \cos \frac{j \omega t}{k}+v_{j, k} \sin \frac{j \omega t}{k}\right) .
\end{gathered}
$$

Meanwhile, for a period- $k$ orbit, if $\phi_{i}(x): S^{1} \rightarrow C[-1,1]$, as in the case of the pendulum, then continuous functions $\phi_{i}\left(x_{k}\right), \phi_{i}\left(x_{k}\right) \cos t$ and $\phi_{i}\left(x_{k}\right) \sin t$ can also be approximated by

$$
\begin{gathered}
\phi_{i}\left(x_{k}\right) \approx \frac{c_{i 0, k}}{2}+\sum_{j=1}^{M}\left(c_{i j, k} \cos \frac{j \omega t}{k}+d_{i j, k} \sin \frac{j \omega t}{k}\right), \\
\phi_{i}\left(x_{k}\right) \cos t \approx \frac{e_{i 0, k}}{2}+\sum_{j=1}^{M}\left(e_{i j, k} \cos \frac{j \omega t}{k}+f_{i j, k} \sin \frac{j \omega t}{k}\right), \\
\phi_{i}\left(x_{k}\right) \sin t \approx \frac{g_{i 0, k}}{2}+\sum_{j=1}^{M}\left(g_{i j, k} \cos \frac{j \omega t}{k}+h_{i j, k} \sin \frac{j \omega t}{k}\right) .
\end{gathered}
$$

Substituting (10)-(14) into (6), the differential equation can be transformed into the following matrix equation for a period- $k$ orbit

$$
\begin{gathered}
\left(\begin{array}{cccccccc}
\omega_{c k} & e_{10, k} / 2 & g_{10, k} / 2 & c_{10, k} / 2 & \cdots & e_{N 0, k} / 2 & g_{N 0, k} / 2 & c_{N 0, k} / 2 \\
\frac{\omega}{k} v_{1, k} & e_{11, k} & g_{11, k} & c_{11, k} & \cdots & e_{N 1, k} & g_{N 1, k} & c_{N 1, k} \\
-\frac{\omega}{k} u_{1, k} & f_{11, k} & h_{11, k} & d_{11, k} & \cdots & f_{N 1, k} & h_{N 1, k} & d_{N 1, k} \\
\vdots & \vdots & \vdots & \vdots & \ddots & \vdots & \vdots & \vdots \\
\frac{M \omega}{k} v_{M, k} & e_{1 M, k} & g_{1 M, k} & c_{1 M, k} & \cdots & e_{N M, k} & g_{N M, k} & c_{N M, k} \\
-\frac{M \omega}{k} u_{M, k} & f_{1 M, k} & h_{1 M, k} & d_{1 M, k} & \cdots & f_{N M, k} & h_{N M, k} & d_{N M, k}
\end{array}\right) \cdot\left(\begin{array}{c}
c_{r} \\
n_{1} \\
p_{1} \\
q_{1} \\
\vdots \\
n_{N} \\
p_{N} \\
q_{N}
\end{array}\right) \\
=\left(\begin{array}{c}
0 \\
\frac{\omega^{2}}{k^{2}} u_{1, k} \\
\frac{\omega^{2}}{k^{2}} v_{1, k} \\
\vdots \\
\frac{M^{2} \omega^{2}}{k^{2}} u_{M, k} \\
\frac{M^{2} \omega^{2}}{k^{2}} v_{M, k}
\end{array}\right) .
\end{gathered}
$$

For simplicity, $A_{k}$ is denoted as the left-hand side matrix,

$$
\lambda=\left(\begin{array}{llllllll}
c_{r} & n_{1} & p_{1} & q_{1} & \cdots & n_{N} & p_{N} & q_{N}
\end{array}\right)^{T},
$$

and

$$
\beta_{k}=\left(\begin{array}{llll}
0 & \frac{\omega^{2}}{k^{2}} u_{1, k} & \frac{\omega^{2}}{k^{2}} v_{1, k} \cdots \frac{M^{2} \omega^{2}}{k^{2}} u_{M, k} & \frac{M^{2} \omega^{2}}{k^{2}} v_{M, k}
\end{array}\right)^{T}
$$


Equation (15) can then be expressed as $A_{k} \lambda=\beta_{k}$.

For multiple periodic orbits, by combining the single periodic orbit matrices together, we can obtain the following matrix equation

$$
A \lambda=\beta,
$$

where

$$
\begin{gathered}
A=\left(\begin{array}{lllll}
A_{1}^{T} & \cdots & A_{k}^{T} & \cdots & A_{K}^{T}
\end{array}\right)^{T}, \\
\beta=\left(\begin{array}{lllll}
\beta_{1}^{T} & \cdots & \beta_{k}^{T} & \cdots & \beta_{K}^{T}
\end{array}\right)^{T},
\end{gathered}
$$

and $\mathrm{A}$ is a $K(2 M+1) \times(3 N+1)$. When $K(2 M+1)>3 N+1$, the matrix can be solved by a least mean square method. The least square solution of (16) is

$$
\lambda=\left(A^{T} A\right)^{-1} A^{T} \beta .
$$

The error of the identification algorithm can be affected by several factors, including accuracy of unstable periodic orbits, noise contamination, system nonlinearity and the choice of basis functions.

\section{Introduction to phase plane reconstruction and extraction of unstable periodic orbits}

Usually in an experiment, only a few signals can be acquired accurately, e. g. angular displacement signal in this simulation. However, for the extraction of unstable periodic orbits, phase plane information is necessary. It is then that the phase plane reconstruction technique [28] is applied. Suppose that $s(k)$ is the sampled smooth signal from dynamical systems, smooth dynamics in a $n$ dimensional phase space could be approximately represented by an embedding dimension space

$$
S(k)=\left\{s(k), s\left(k+T_{d}\right), \ldots, s\left(k+(d-1) T_{d}\right)\right\},
$$

where $d$ is the dimension of the reconstructed phase space and $T_{d}$ is the time delay of the embedding dimension. Although the reconstructed phase space is a distorted appearance of the real phase plane, it provides us information of phase orbits, attractors, periodic orbits and other chaotic characteristics. In the pendulum system, $s(k)=\theta(k), \theta \in(-\pi, \pi)$ is actually a non-smooth observable. But the method can also be applied here since the functions of angular displacement, speed and acceleration are all smooth. 


\subsection{Choosing the embedding Dimension and time Delay}

The time delay value $T_{d}$ can be obtained from average mutual information function [28], which is expressed as

$$
I_{A B}(t)=\sum_{a_{i}, b_{k}} P_{A B}\left(a_{i}, b_{k}\right) \log _{2} \frac{P_{A B}\left(a_{i}, b_{k}\right)}{P_{A}\left(a_{i}\right) P_{B}\left(b_{k}\right)},
$$

where $t$ is the time lag, $a_{i}$ is the sampled data $s(i), A$ is the set of $\{s(i)\}, k=i+T, b_{k}$ is thus $s(i+t)$, and $B$ is considered as the set of $\{s(i+t)\}$. Meanwhile, $P_{A}(x)$ is the probability function of observing $x$ out of a set $A, P_{B}(y)$ is the probability of observing $y$ out of a set $B$, and $P_{A B}(x, y)$ is the joint probability function of observing $x$ and $y$ out of $A$ and $B$. After calculating $I(t)$, the best time delay $T_{d}$ is chosen when $I(t)$ reaches its first minimum. To determine the value of $d_{E}$ for minimum required delay dimensions, the false nearest neighborhood method was applied [28].

\subsection{Extracting Unstable Periodic Orbits (UPOs)}

There are numerous unstable periodic orbits in a chaotic signal. The reconstructed phase space can be used to extract the hidden UPOs. Since the horizontally excited single pendulum is excited by a harmonic signal with period $T$, the UPOs will have the periodicity of integer multiples of the excitation period $T$. Though theoretically, no exact UPO can be found through the collected data, the theory proved that some very close approximation of the UPO exists provided that the data is long enough. With the error tolerance $e$ set for UPO extraction, we say that a approximated UPO of period $k$ is extracted if $|S(n-k T)-S(n)|_{c}<e$. Noticing that in $S^{1}$ space, the distance between two points, $x$ and $y$, is measured on a circle, and thus can be expressed as

$$
|x-y|_{c}=|(\pi+|x-y| \bmod 2 \pi)-\pi| .
$$

Usually $e$ is set as $1-5 \%$ of the span of the data [26], and the smaller $e$ value is always desirable if possible, since the corresponding UPOs will be closer to the real periodic orbits.

\section{Numerical simulation of the horizontally excited pendulum}

\subsection{Phase Plane ReCONStRUCtion}

The simulation was based upon the non-dimensional differential equation (1). A data set of 30000 points was gathered with a sampling rate $f_{s}=25 / T$. Displayed in Figure 2 (a) is the 2-D reconstructed phase space. The coefficients were chosen as $f=1.52, c=0.03, r=0.8333$. The actual minimum embedding dimension needed for a complete phase plane reconstruction was found to be 


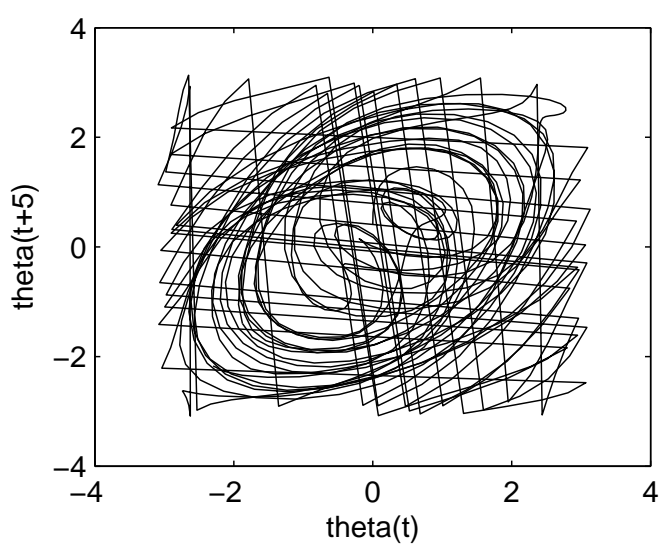

(a)

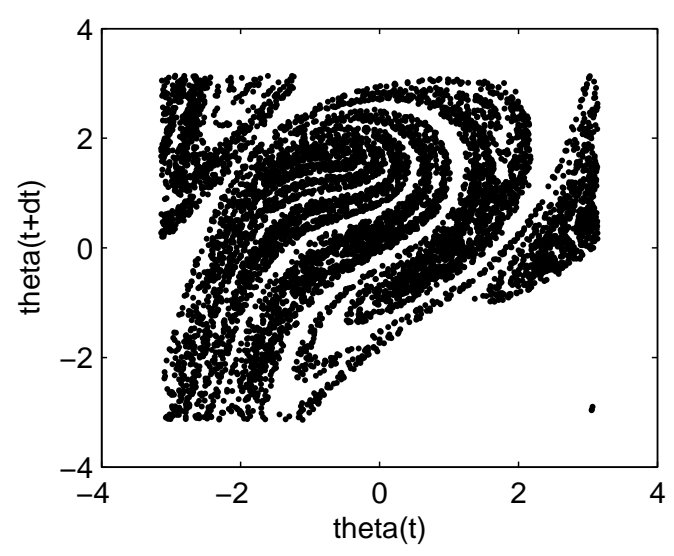

(b)

Figure 2. Reconstructed phase plane (a) and Poincaré section plot (b); $\theta(t)$ is represented by theta(t) in the plots.

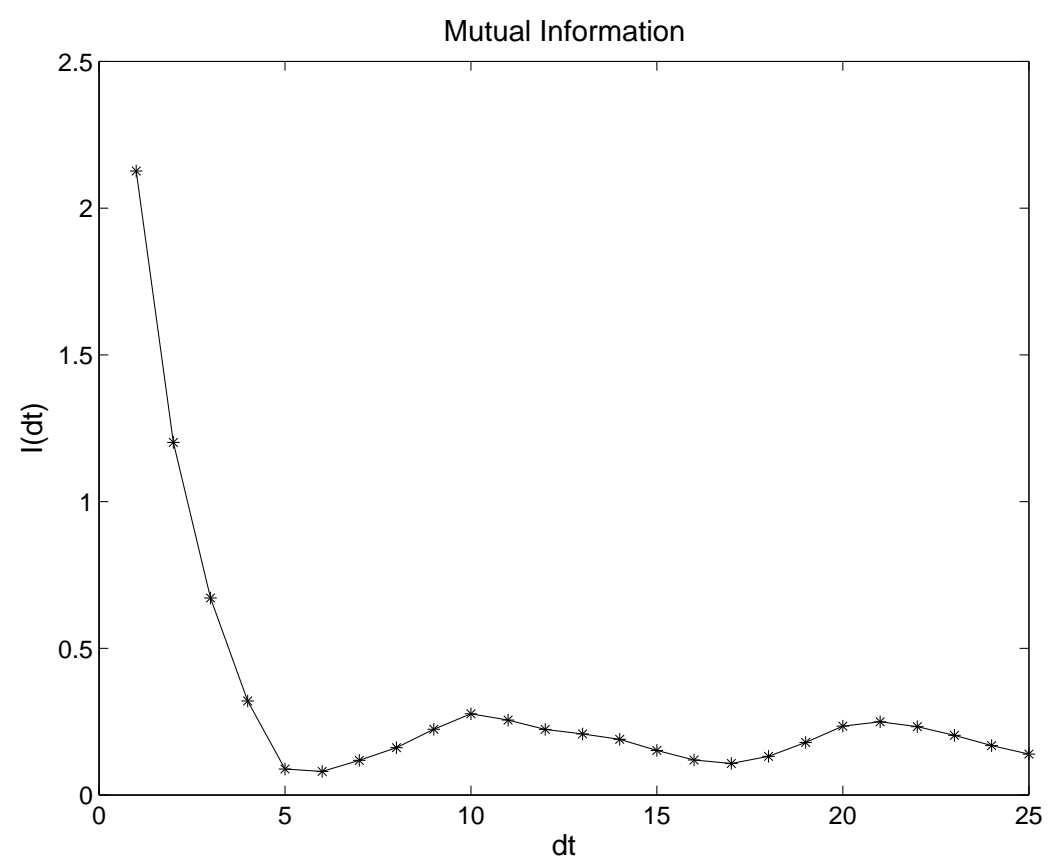

Figure 3. Mutual information of the data; the first minimum is at $d t=5$.

four. However, since our purpose of reconstructing phase plane is to extract UPOs, which compare points with $k T$ time interval (implying the addition of the $S^{1}$ dimension of time), the possibility of false nearest neighbor is then greatly reduced. Hence, two embedding coordinates are adequate for this case, and also simpler for display purposes.

Meanwhile, according to Figure 3 of average mutual information, the calculated best time delay was $T_{d}=5$. The Poincaré section is displayed in Figure $2(\mathrm{~b})$. The Poincaré section plot provides us 


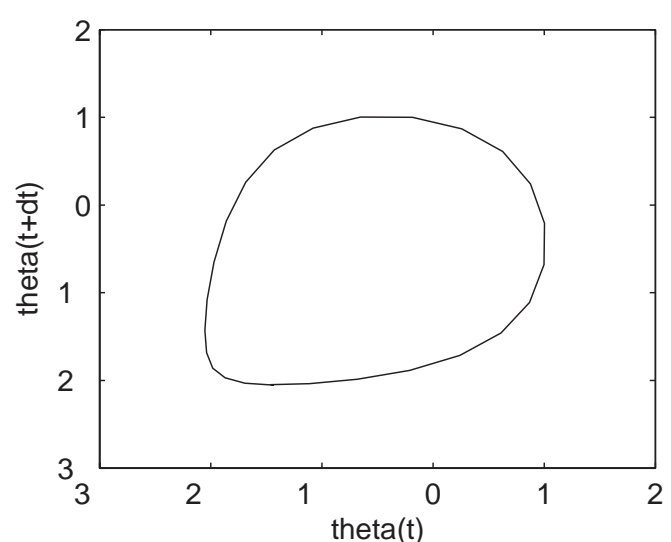

(a)

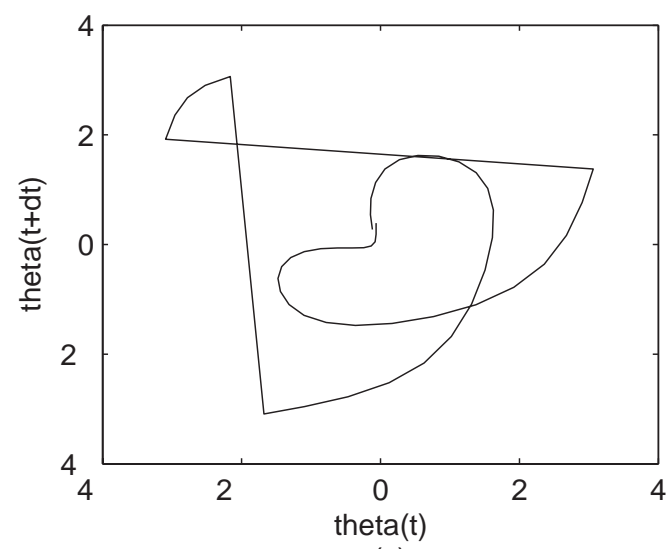

(c)

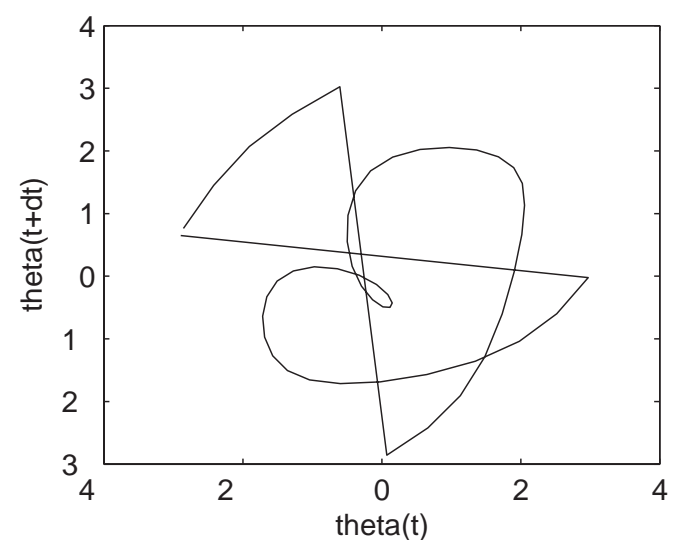

(b)

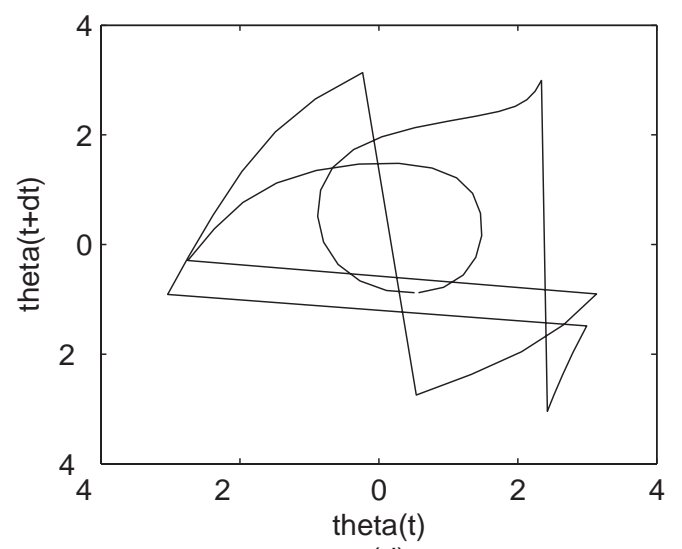

(d)

Figure 4. (a)A period-1 orbit; (b) A period-2 orbit; (c) A period-2 orbit; (d) A period-2 orbit.

a handy tool to visually compare chaotic properties of different systems. Similar Poincaré section plots give evidence of similar dynamical behaviors of the systems.

\subsection{PARAMETRIC IDENTIFICATION}

For simplicity, the data used in identification process was noise-free, thus excluding the noisegenerated error in the identified parameters. The UPOs were extracted before applying identification algorithm. An error tolerance of $e=3 \%$ was applied in the extraction. The error tolerance can be made smaller if longer sampled data is available. Consequently, around 25 UPOs were extracted from period 1 to period 16. Figure 4 shows a example of periodic orbits of period one and two. Some are whirling orbits, whereas others are oscillating orbits. With the UPOs extracted, in the identification process, two sets of basis functions were tested to approximate the unknown nonlinear functions in the governing equation. 
Table I. $n_{k}, p_{k}$ and $q_{k}$ values when harmonic basis is applied.

\begin{tabular}{|c|c|c|c|c|c|c|c|c|c|}
\hline- & $\hat{n}_{k}$ & $n_{k}$ & $n_{k}$ error & $\hat{p}_{k}$ & $p_{k}$ & $p_{k}$ error & $\hat{q}_{k}$ & $q_{k}$ & $q_{k}$ error \\
\hline $\cos \theta$ coeff. & 0.0093 & 0.0 & 0.0093 & -1.5255 & -1.52 & 0.0055 & -0.0044 & 0.0 & 0.0044 \\
\hline $\sin \theta$ coeff. & 0.0034 & 0.0 & 0.0034 & -0.0 & 0.0 & 0.0 & 1.4335 & $1.44 \mid 0.0065$ \\
\hline
\end{tabular}

\subsubsection{Harmonic basis functions}

If basis functions are set as $\phi_{2 k-1}(x)=\cos k x, \phi_{2 k}(x)=\sin k x, k=1,2,3, \ldots$, the differential equation (6) is then converted to

$$
\begin{aligned}
c_{r} \dot{x} & +\sum_{k=1}^{K}\left(n_{2 k-1} \cos k x+n_{2 k} \sin k x\right) \cos t+\sum_{k=1}^{K}\left(p_{2 k-1} \cos k x+p_{2 k} \sin k x\right) \sin t \\
& +\sum_{k=1}^{K}\left(q_{2 k-1} \cos k x+q_{2 k} \sin k x\right)=-\ddot{x}
\end{aligned}
$$

For the investigated pendulum, it is convenient to set $K=1$, i. e. only the first harmonics, since the differential equation is simple and consists of harmonic type non-linearity.

For systems like the single pendulum, where the non-linearity comes from angular rotation and the displacement variable belongs to the sphere space, a harmonic basis can be a very good choice due to its capability of representing rotation and periodic behaviors. Displayed in Table I are the identified coefficients of $(20)$ with $\hat{n}_{k}, \hat{p}_{k}, \hat{q}_{k}$ representing the estimated values, and $n_{k}, p_{k}$, $q_{k}$ representing the true values. The estimated $c_{r}$ value is 0.0339 . Compared to the real values, all of the parameters are quite accurate with minor errors less than 0.01 .

\subsubsection{Linear interpolation basis functions}

Linear interpolation functions can be expressed as

$$
\phi_{k}(x)=\left\{\begin{array}{ll}
\frac{x-(k-1) d}{d}, & (k-1) d<x<k d \\
\frac{(k+1) d-x}{d}, & k d \leq x<(k+1) d \\
0, & \text { otherwise }
\end{array},\right.
$$

where $d$ is the distance interval. Had we not known the appropriate basis functions, e. g. harmonics in this case, interpolation functions are good alternatives [18]. For better expression of the nonlinear functions, generally more interpolation points (functions) are needed [27]. However, that implies more unknown parameters and requires more periodic orbits for the least mean square method. Eight points were utilized in this identification. The interval $[-\pi, \pi)$ was divided evenly into eight equal sub-intervals. The unknown curves can be represented by the piece-wise linear curve connected between sub-interval nodes.

Figures 5, 6, and 7 show the piece-wise curves of $\left\{n_{k}\right\},\left\{p_{k}\right\},\left\{q_{k}\right\}$. Meanwhile, the identified friction coefficient $c_{r}=0.0348$. It can be seen that $\left\{n_{k}\right\}$ curve is nearly zero, $\left\{p_{k}\right\}$ curve is a 


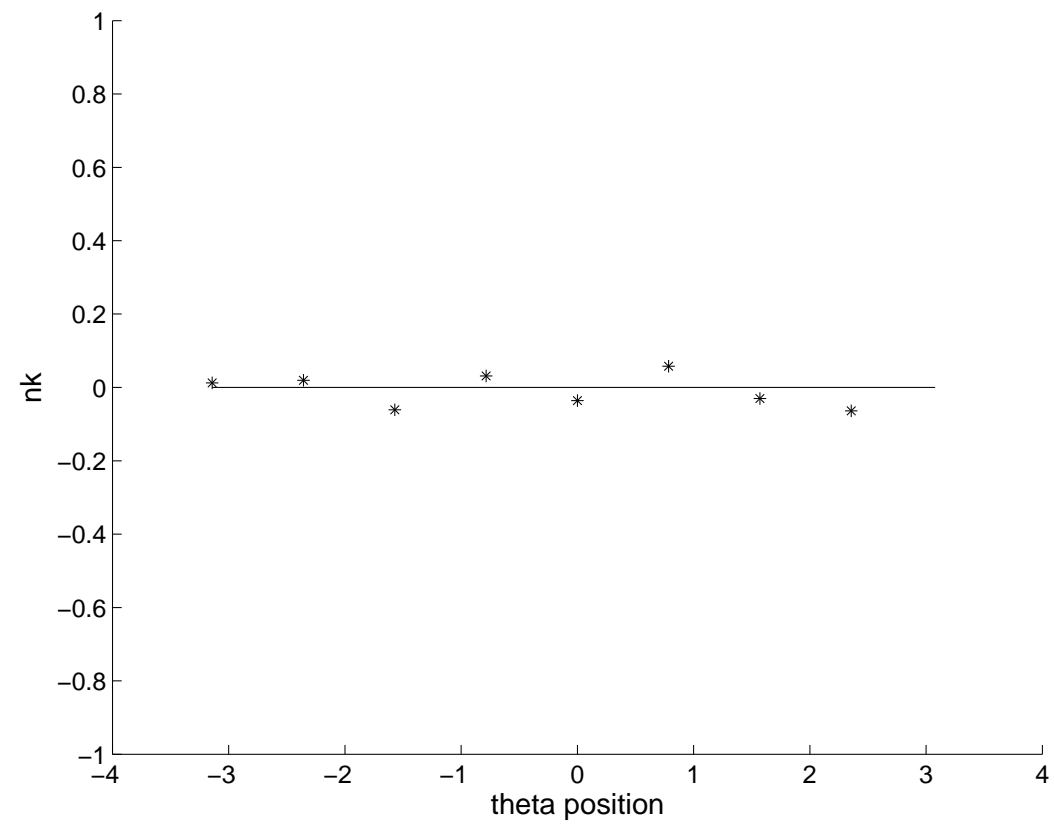

Figure 5. $n_{k}$ curve for linear interpolation basis; $\left(^{*}\right)$ identified values, $(-)$ true function.

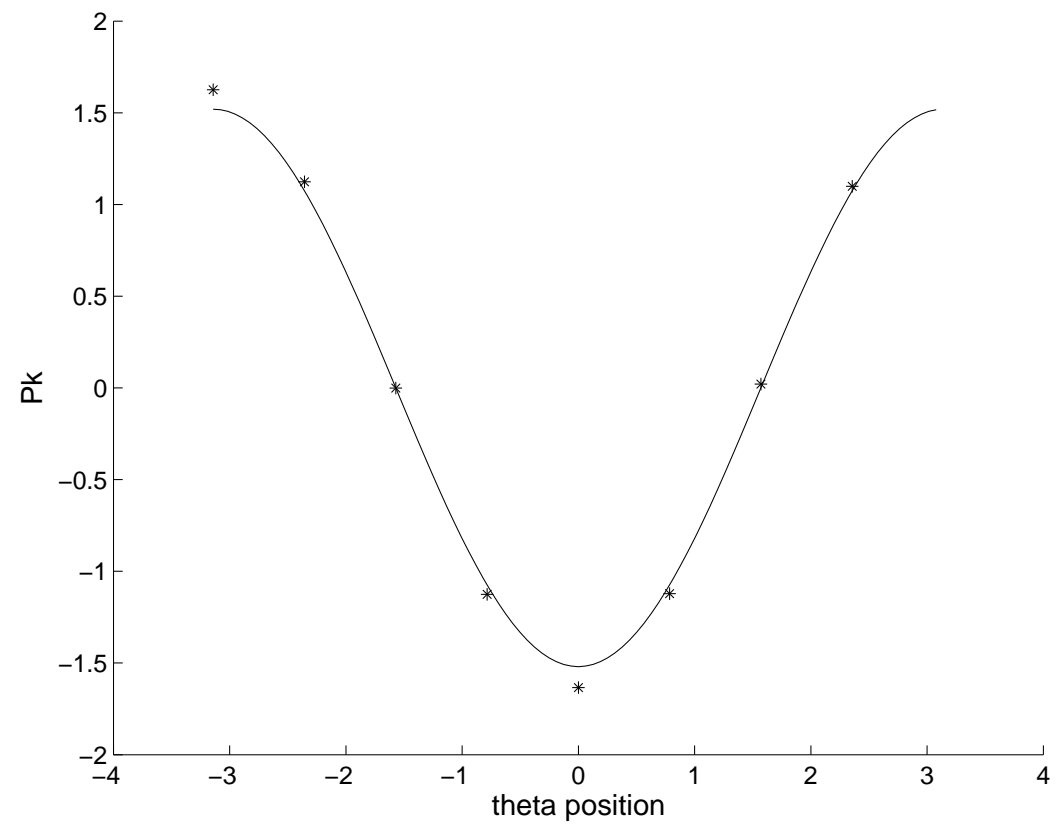

Figure 6. $p_{k}$ curve for linear interpolation basis; $(*)$ identified values, $(-)$ true function. 


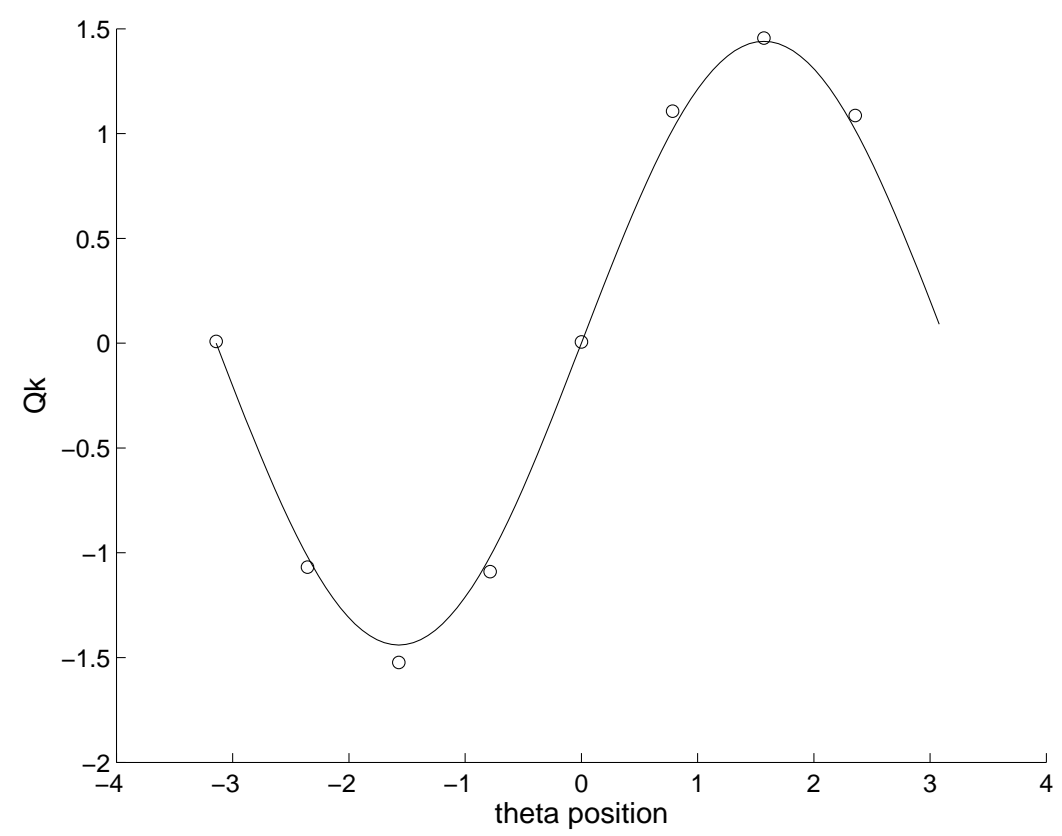

Figure 7. $q_{k}$ curve for linear interpolation basis; (o) identified values, $(-)$ true function.

cosine curve, and the $\left\{q_{k}\right\}$ curve is a sine curve, which are consistent with the differential equation (1). However, eight points are still a rough representation of the real curve, and consequently the interpolation functions introduces more error than the harmonic basis case. In the next section, we will discuss the error's influence on the dynamic characteristics of the system.

\section{Discussion and Validation}

Direct comparison showed that the identification algorithm was satisfying for the single degree of freedom system. Furthermore, it was the harmonic basis that generated the more precise identification result, due to the fact that the unknown nonlinear functions consist of harmonics. However, when investigating unknown systems, direct comparison is unavailable. In this case, it is then natural to examine the dynamical behavior, e. g. chaotic characteristics, of the identified system for validation purposes.

Displayed in Figure 8 (a) and (b) are Poincaré sections of the identified systems with harmonic basis and linear interpolation basis. The Poincaré sections look similar to the original pendulum, which implies the dynamical similarity of these three chaotic systems. Thus, for the description of chaotic characteristics, the linear interpolation approximation, though a coarse representation, is still acceptable. 


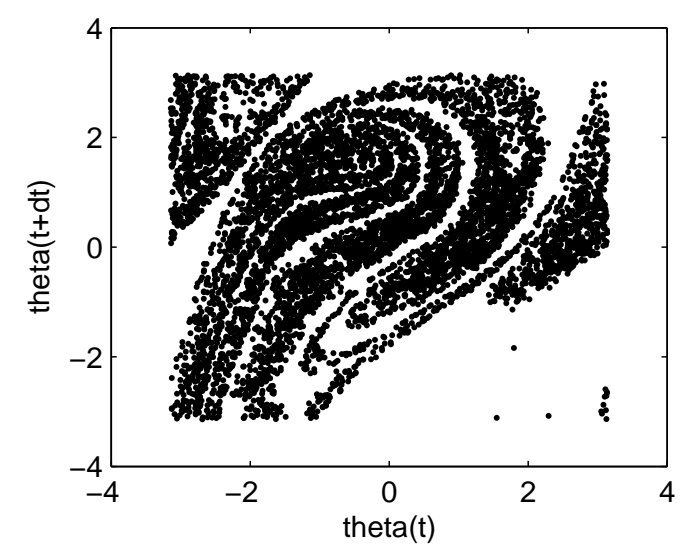

(a)

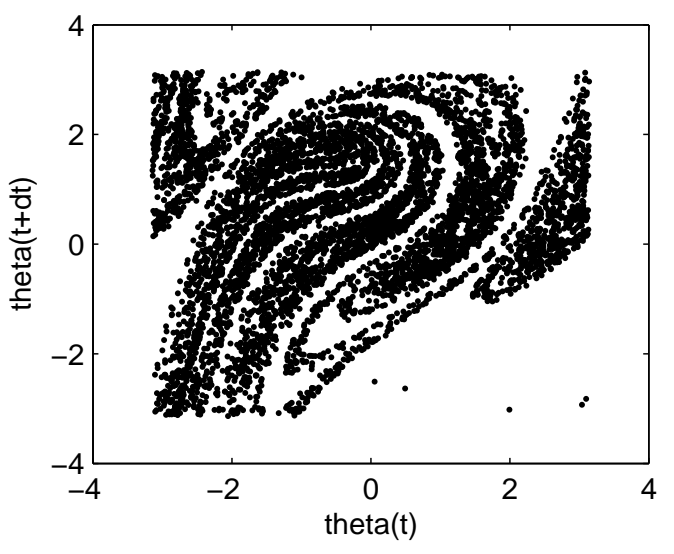

(b)

Figure 8. Poincaré section plots of identified systems: (a) by linear interpolation basis, (b) by Harmonic basis.

For more validation methods of the unknown systems, we can also refer to the linearized model, comparison of fractal dimensions, vector fields [27], bifurcation diagrams, and Lyapunov exponents. But for this simulation case, where the parameters are known, direct comparison of parameters is the best tool.

\section{Conclusions}

In this paper, the simulated parametric identification procedure was investigated on a chaotic system of a horizontally base excited pendulum. Theoretically, the identification algorithm can be applied to single degree of freedom system with a known excitation frequency and unknown parametric, or forced excitation functions. The identification algorithm [26, 27] was modified and improved for application to the whirling pendulum system with parametric excitation. Two types of basis functions were applied to approximate the unknown functions. Then, the simulations of the identified models were presented to verify the effectiveness of the algorithm.

The direct comparison of both parameters and Poincaré sections showed the accuracy of the identified parameters and the similarity between the original and the identified systems. Although both of the methods gave similar results, the harmonic basis functions matched the form of the true nonlinearity, and therefore in this case, gave a more precise result. On the other hand, the linear interpolation was also shown to be valuable since it is a good choice for a general unknown type of non-linearity in a single variable.

According to the simulation result, the improved parametric identification process was successful in single degree-of-freedom parametrically excited whirling systems. It is also promising to apply 
this method to more general dynamic systems, such as rotational and/or multi-degree-of-freedom systems. For this study, the simulation was limited to single degree of freedom systems with no noise contamination. Hence, the error and convergence analysis was not addressed in this study. However, we do know that to obtain more accurate identification results, more accurate UPOs are necessary, i. e. small recurrence error in the UPO extraction. For more general applications, multi-degree of freedom systems and experimental verifications are needed for full investigation of the proposed method.

\section{Acknowledgements}

We would like to express our appreciation to NASA (grant number NAG-1-01048, with Dr. Walt Silva) for supporting this research.

\section{References}

1. Willem van de Water, Marc Hoppenbrouwers, 'Unstable periodic orbits in the parametrically excited pendulum', Physical Review A, Vol. 44(10), 1991, pp. 6388-6397.

2. Jaeyong Jeong, Sang-Yoon Kim, 'Bifurcations in a Horizontally Driven Pendulum', Journal of Korean Physical Society, Vol. 35(5), 1999, pp. 393-398.

3. S. R. Bishop, M. J. Clifford, 'Zones of Chaotic Behavior in the parameterically excited pendulum', Journal of Sound and Vibration, Vol. 189, 1996, pp. 142-147.

4. R. V. Dooren, 'Chaos in a pendulum with forced horizontal support motion: a tutorial', Chaos, Solutions ES Fractals, Vol. 7(1), 1996, pp. 77-90.

5. V. B. Raybov, 'Using Lyapunov exponents to predict the onset of chaos in nonlinear oscillators', Physical Review E, Vol. 66(1), No. 016214, Part 2, 2002.

6. W. Szemplinska-Stupnicka, E. Tyrkiel, 'The oscillation-rotation attractors in the forced pendulum and their peculiar properties', International Journal of Bifurcation and Chaos, Vol. 12(1), 2002, pp. 159-168.

7. M. Clerc, P. Coullet, E. Tirapegui, 'The stationary instability in quasi-reversible systems and the Lorenz pendulum', International Journal of Bifurcation and Chaos, Vol. 11(3), 2001, pp. 591-603.

8. K. S. Mohammad and et al., 'Direct parameter estimation for linear and non-linear structures,' Journal of Sound and Vibration, vol. 153(3), 1992, pp. 471-499.

9. Q. Chen, G. R. Tomlinson, 'Parametric identification of systems with dry friction and nonlinear stiffness a time series model', Journal of Vibration and Acoustics, Vol. 118 (2), 1996, pp. 252-263.

10. N. P. Plakhtienko, 'Methods of identification of nonlinear mechanical vibrating systems', International Applied Mechanics, Vol. 36 (12), 2000, pp. 1565-1594

11. N. P. Plakhtienko, 'A method of special weight-functions in the problem of parametric identification of mechanical systems', DOPOV AKAD NAUK A, Vol. 8, 1983, pp. 31-35.

12. R. K. Kapania, and S. Park, 'Parametric identification of nonlinear structural dynamic systems using time finite element method', AIAA Journal, Vol. 35 (4), 1997, pp. 719-726

13. G. Verbeek, A. Dekraker, D. H. Vancampen, 'Nonlinear parametric identification using periodic equilibrium states - application to an aircraft landing gear damper', Nonlinear Dynamics, Vol. 7 (4), 1995, pp. 499-515.

14. M. R. Hajj, J. Fung, A. H. Nayfeh, et al. , 'Parametric Identification of Nonlinear Dynamic Systems', Computers ES Structures, Vol. 20 (3), 1985, pp. 487-493.

15. K. Yasuda, S. Kawamura, and K. Watanabe, 'Identification of nonlinear multi-degree-of-freedom systems (presentation of a technique)', JSME International Journal, Series III, Vol 31, 1988, pp. 8-14. 
16. K. Yasuda, S. Kawamura, 1989, 'A nonparametric identification technique for nonlinear vibratory systems (proposition of a technique)', JSME International Journal, Series III, Vol 31, 1989, pp. 365-372.

17. K. Yasuda, K. Kamiya, 'Identification of a nonlinear beam (proposition of an identification technique)', JSME International Journal, Series III, Vol 33, 1990, pp. 535-540.

18. K. Yasuda, K. Kamiya, 'Experimental identification technique of nonlinear beams in time domain', Nonlinear Dynamics, Vol. 18, 1997, pp. 185-202.

19. G. Kerschen, V. Lenaerts, J. C. Golinval, 'Identification of a continuous structure with a geometrical nonlinearity. Part I: Conditioned reverse path method', Journal of Sound and Vibration, Vol. 262(4), 2003, pp. 889906.

20. C. M. Richard, R. Singh, 'Identification of multi-degree-of-freedom non-linear systems under random excitations by the reverse-path spectral method', Journal of Sound and Vibration, Vol. 213(4), 1998, pp. 673-708.

21. Michael Feldman, 'Non-linear system vibration analysis using Hilbert transform-I. free vibration analysis method "FREEVIB" ', Mechanical Systems and Signal Processing, Vol. 8(2), 1994, pp. 119-127.

22. V. Lenaerts, G. Kerschen, J. -C. Golinval, 'Identification of a continuous structure with a geometrical nonlinearity. Part II: Proper orthogonal decomposition', Journal of Sound and Vibration, Vol. 262(4), 2003, pp. 907919.

23. G. Kerschen, B. F. Feeny, J. C. Golinval, 2003, 'On the exploitation of chaos to build reduced-order models', Computer Methods in Applied Mechanics and Engineering, Vol. 192(13-14), 2003, pp. 1785-1795.

24. M. Feldman, 1997, 'Non-linear free vibration identification via the Hilbert transform', Journal of Sound and Vibration, Vol. 208(3), 1997, pp. 475-489.

25. Roger Ghanem, F. Romeo, 2001, 'A wavelet-based approach for model and parameter identification of nonlinear systems', International Journal of Non-linear Mechanics, Vol. 36, 2001, pp. 835-859.

26. C. M. Yuan, B. F. Feeny, 'Parametric Identification of Chaotic Systems', Journal of Vibration and Control, Vol. 4(4), 1998, pp. 405-426.

27. B. F. Feeny, C. M. Yuan, J. P. Cusumano, 'Parametric identification of an experimental magneto-elastic oscillator', Journal of Sound and Vibration, Vol. 247(5), 2001, pp. 785-806.

28. H. D. I. Abarbanel, R. Brown, J. J. SIDorowich, L. S. Tsimring, 'The analysis of observed chaotic data in physical systems', Reviews of Modern Physics, Vol. 65, 1993, pp. 1331-1392. 